\title{
Particle identification algorithms for the PANDA Barrel DIRC
}

\author{
A. Ali, ${ }^{a, b}, 1$ A. Belias, ${ }^{a}$ R. Dzhygadlo, ${ }^{a}$ A. Gerhardt, ${ }^{a}$ M. Krebs, ${ }^{a, b}$ D. Lehmann, ${ }^{a}$ K. Peters, ${ }^{a, b}$ \\ G. Schepers, ${ }^{a}$ C. Schwarz, ${ }^{a}$ J. Schwiening, ${ }^{a}$ M. Traxler, ${ }^{a}$ L. Schmitt, ${ }^{c}$ M. Böhm, ${ }^{d}$ \\ A. Lehmann, ${ }^{d}$ M. Pfaffinger, ${ }^{d}$ S. Stelter, ${ }^{d}$ M. Düren, ${ }^{e}$ E. Etzelmüller, ${ }^{e}$ K. Föhl, ${ }^{e}$ \\ A. Hayrapetyan, ${ }^{e}$ I. Köseoglu, ${ }^{e, a}$ K. Kreutzfeld, ${ }^{e}$ M. Schmidt, ${ }^{e}$ T. Wasem, ${ }^{e}$ C. Sfienti, ${ }^{f}$ \\ A. Barnyakov, ${ }^{g}$ K. Beloborodov, ${ }^{g}$ V. Blinov, ${ }^{g}$ S. Kononov, ${ }^{g}$ E. Kravchenko ${ }^{g}$ and I. Kuyanov ${ }^{g}$ \\ ${ }^{a}$ GSI Helmholtzzentrum für Schwerionenforschung GmbH, Darmstadt, Germany \\ ${ }^{b}$ Goethe University, Frankfurt a.M., Germany \\ ${ }^{c}$ FAIR, Facility for Antiproton and Ion Research in Europe, Darmstadt, Germany \\ ${ }^{d}$ Friedrich Alexander-University of Erlangen-Nuremberg, Erlangen, Germany \\ ${ }^{e}$ II. Physikalisches Institut, Justus Liebig-University of Giessen, Giessen, Germany \\ ${ }^{f}$ Institut für Kernphysik, Johannes Gutenberg-University of Mainz, Mainz, Germany \\ ${ }^{g}$ Budker Institute of Nuclear Physics of Siberian Branch Russian Academy of Sciences, Novosibirsk, Russia \\ E-mail: a.ali@gsi.de
}

AbstRact: The PANDA experiment at the international accelerator Facility for Antiproton and Ion Research in Europe (FAIR) near GSI, Darmstadt, Germany will address fundamental questions of hadron physics. Excellent particle identification is required to achieve the PANDA physics goals. Hadronic particle identification (PID) in the barrel region of PANDA target spectrometer will be delivered by a fast focusing DIRC (Detection of Internally Reflecfted Cherenkov light) counter. The Barrel DIRC will cover the polar angle range of $22^{\circ}-140^{\circ}$ and is designed to provide $\pi / \mathrm{K}$ separation for momenta up to $3.5 \mathrm{GeV} / c$ with a separation power of at least 3 standard deviations. Several reconstruction algorithms have been developed to determine the performance of the detector. The "geometrical reconstruction" determines the Cherenkov angle by relying primarily on the position of the detected photons. The "time imaging", however, utilizes both position and time measurements by directly performing the maximum likelihood fit. Simulations and experimental data from prototype tests at the CERN Proton Synchrotron (PS) were used to evaluate the performance of the algorithms. We will discuss both reconstruction approaches.

Keywords: Cherenkov detectors; Detector modelling and simulations I (interaction of radiation with matter, interaction of photons with matter, interaction of hadrons with matter, etc); Particle identification methods; Performance of High Energy Physics Detectors

${ }^{1}$ Corresponding author. 


\section{Contents}

1 Introduction 1

2 Prototype setup 1

3 Prototype simulation 3

4 Comparison of reconstruction algorithms 4

4.1 Geometrical Reconstruction 4

4.1.1 Cherenkov angle data PDF $\quad 8$

4.2 Time-based imaging 9

$\begin{array}{lll}4.3 & \text { Simplified time-based imaging } & 11\end{array}$

5 Conclusion 12

\section{Introduction}

The PANDA Barrel DIRC [1] will play a crucial role in the success of the high luminosity PANDA experiment at FAIR by providing clean particle identification (PID) for charged hadrons with momenta up to $3.5 \mathrm{GeV} / \mathrm{c}$ in the polar angle range of $22^{\circ}$ to $140^{\circ}$ with at least 3 standard deviations separation power [2]. Several algorithms were developed to reconstruct the Cherenkov angle and to perform PID. These algorithms were applied to detailed Geant 4 simulations and beam test data from a prototype at the CERN PS to compare the performance.

\section{Prototype setup}

During the most recent beam test campaign, in July/August 2018, the prototype was placed into the mixed hadron beam in the T9 experimental zone at the CERN PS. The T9 beam was primarily composed of electrons, muons, pions, and protons and had a very low kaons flux. Since the direct evaluation of $\pi / \mathrm{K}$ separation at $3.5 \mathrm{GeV} / c$ was not feasible, the PID performance was evaluated for $\pi / \mathrm{p}$ at $7 \mathrm{GeV} / c$ instead. At this momentum the Cherenkov angle difference between pions and protons is equivalent to pions and kaons at $3.5 \mathrm{GeV} / c$, the upper limit of the PID performance range of the PANDA Barrel DIRC.

A CAD drawing of the experimental setup in the T9 hall can be seen in figure 1. The Barrel DIRC prototype was placed between two time-of-flight (TOF) detectors that were spaced $29 \mathrm{~m}$ apart and used for $\pi / \mathrm{p}$ tagging. A scintillating fiber hodoscope delivered charged track position information. Figure 2 shows the clear separation of pions and protons by the TOF system for a beam momentum of $7 \mathrm{GeV} / c$. Three scintillator counters (Trigger 1, Trigger 2 Vertical, and Trigger 2 Horizontal in figure 1) were placed in front of and behind the prototype. 


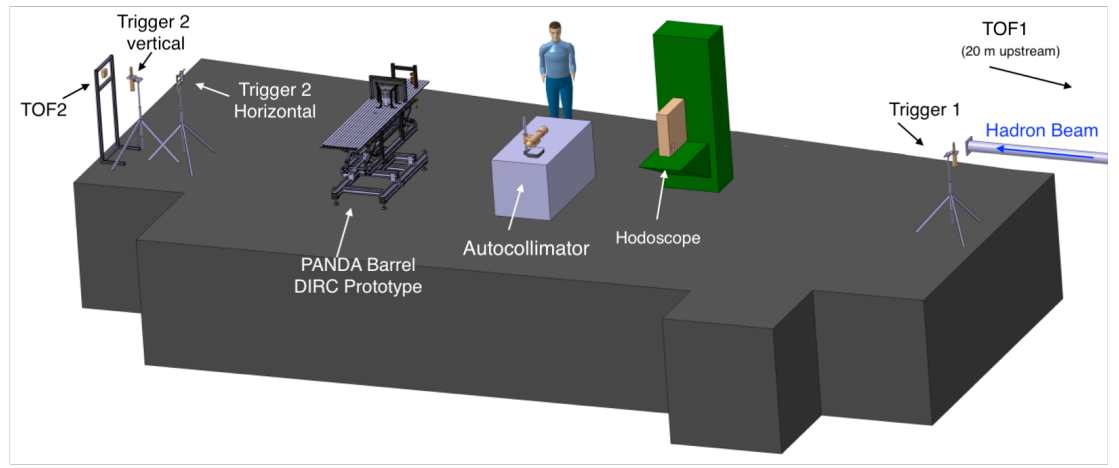

Figure 1. CAD drawing of the T9 experimental hall with the PANDA DIRC prototype setup. Two time-offlight (TOF) detectors were separated by $29 \mathrm{~m}$ and used as external $\pi / \mathrm{p}$ identification.

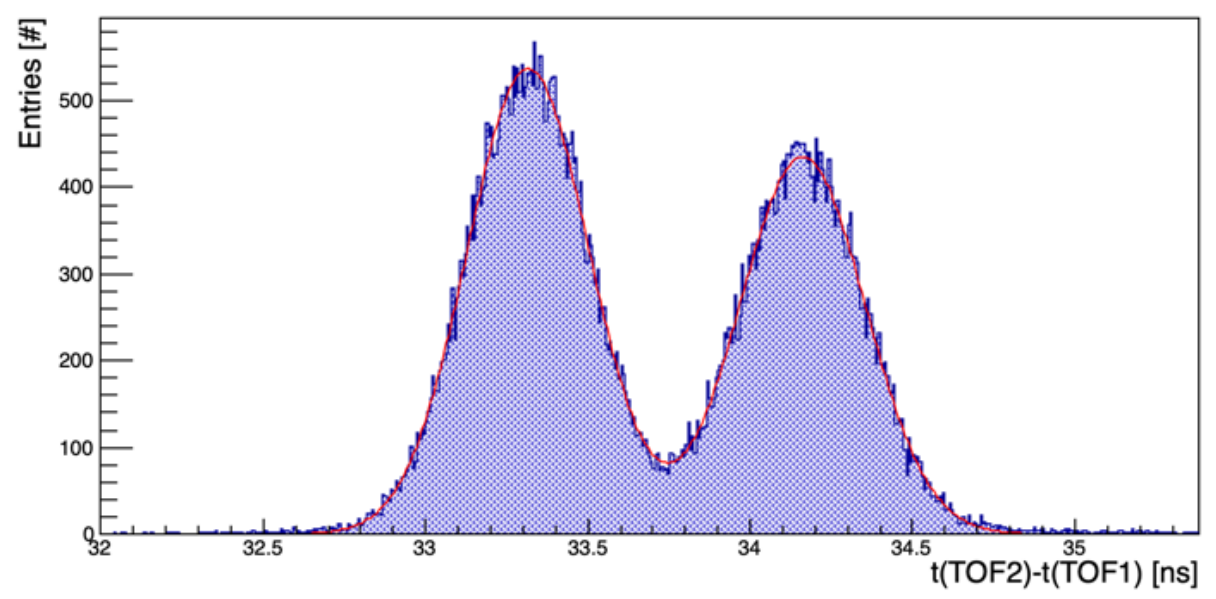

Figure 2. $\pi / \mathrm{p}$ tagging provided by the time-of-flight system at $7 \mathrm{GeV} / c$ momentum and $29 \mathrm{~m}$ distance between TOF1 and TOF2.

A coincidence of the trigger signals was used by the Data Acquisition (DAQ) system for event recording.

Cherenkov photons, emitted along the charged particle track in a fused silica bar, are transported via total internal reflection inside the bar. A mirror is attached to one end of the bar to reflect photons towards the other end, where a 3-layer spherical lens focuses the photons on the back of a fused silica prism with $300 \mathrm{~mm}$ depth and an opening angle of $33^{\circ}$, serving as expansion volume.

A $2 \times 4$ array of PHOTONIS Planacon Microchannel Plate Photomultiplier Tubes (MCPPMTs) [3] with a total of 512 pixels $\left(6 \times 6 \mathrm{~mm}^{2}\right.$ each $)$ is attached to the prism and held in place by a 3-D printed support structure. The optical coupling between the bar/lens, lens/prism, and prism/MCP-PMTs was made using Eljen EJ-550 optical grease [4]. 


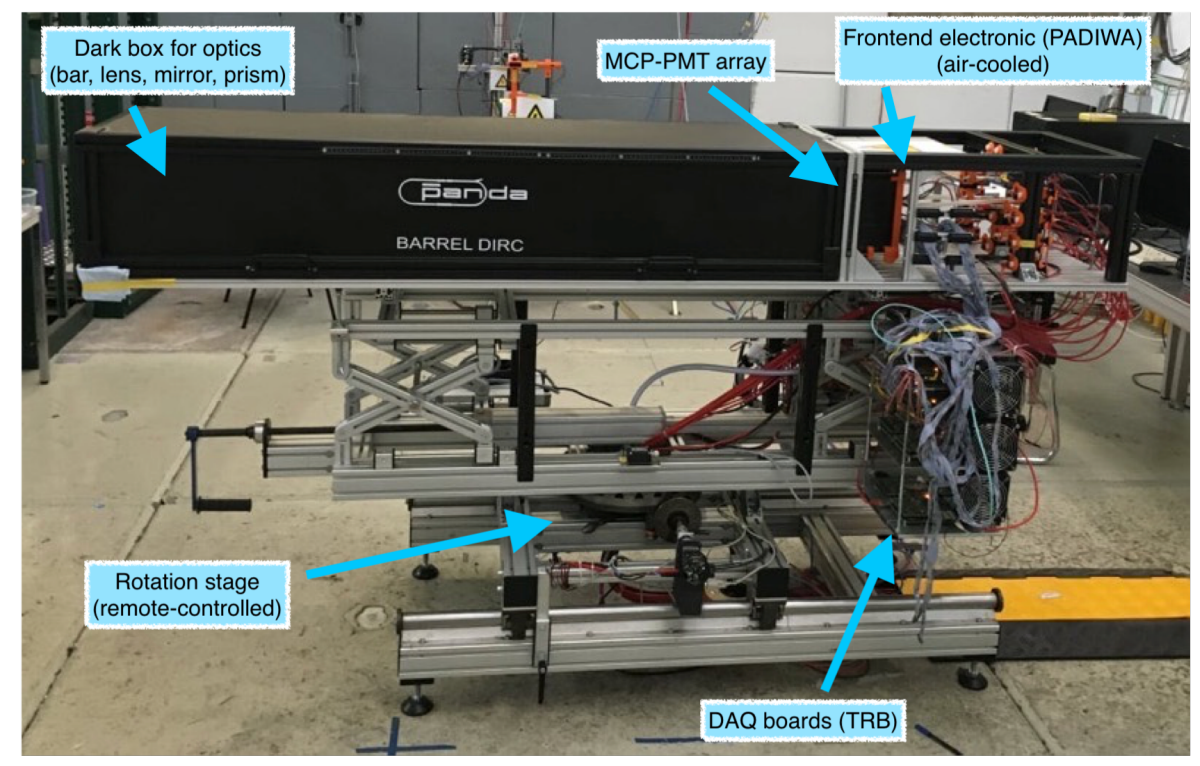

Figure 3. Photo of the PANDA Barrel DIRC prototype in the T9 beamline at CERN.

The MCP-PMTs were read out by a DAQ system based on the HADES readout board TRB3 and the PADIWA discriminator card [5], recording the arrival time of the photons and the timeover-threshold of the signal. Background from dark noise of the sensors and signals from delta electrons were removed using the timing information.

The mechanical system of the prototype was designed with a rotating table and rails that allow the detector to be rotated and translated relative to the beamline. The prototype rotation was remote-controlled and monitored by a camera. The radiator was carefully held in place by a pair of 3-D printed plastic braces equipped with screws, which allowed fine adjustments of the bar position. The optical components of the prototype were covered by a black box to make the setup light-tight. The alignment of all components of the prototype at T9 was performed by an autocollimator and a laser alignment system.

The calibration of each readout channel was performed using a $405 \mathrm{~nm}$ Picosecond Injection Laser (PiLas) [6]. Single-mode optical fibers transported the light to two optical diffusers to evenly illuminate the entire MCP-PMT plane. The data were taken during approximately 4 weeks in the summer of 2018, accumulating roughly one billion triggers, including scans of the polar and azimuthal angles at different momentum points with several geometrical configurations.

\section{Prototype simulation}

The prototype simulation was an important element for the success of the beam test at CERN. During the planning phase, the simulation was used to optimize the MCP-PMT layout on the prism focal plane and to choose the optimum locations of the optical diffusers for the laser calibration. Furthermore, the simulation was performed during the data analysis phase to generate the look-up tables (LUT) for the geometrical reconstruction. A Geant4 [7] stand-alone simulation was developed for the beam test, incorporating many components of the PANDA Geant4 simulation [8], utilizing the 
same physics processes and materials properties tables. The beam properties were included in the simulation as well. For each prototype configuration, the geometry of each optical component (e.g., the orientation of the bar relative to the lens and the prism) was adjusted to the values measured during periods of access to the prototype. Furthermore, the detailed specifications and characteristics of the Planacon MCP-PMTs used during this beam test were included in the simulation. This included the experimentally observed dark count rate, charge-sharing, collection efficiency, and the quantum efficiency as a function of the photon wavelength. The simulated single photon timing precision of the electronics was tuned to match the observed performance in the experiment.

\section{Comparison of reconstruction algorithms}

Several reconstruction algorithms were developed for the PANDA Barrel DIRC. Geant4 simulation of the prototype and CERN PS test beam data were used for testing and performance evaluation of the reconstruction.

\subsection{Geometrical Reconstruction}

The geometrical reconstruction method is based on the successful BaBar DIRC [9] detector reconstruction approach. The method combines the known spatial position of the photon hit at the detection plane and location and direction of the charged particle track in the bar and transforms them into a coordinate system of $\left(\theta_{c}, \phi_{c}, t_{\text {propagation }}\right)$.

(a)

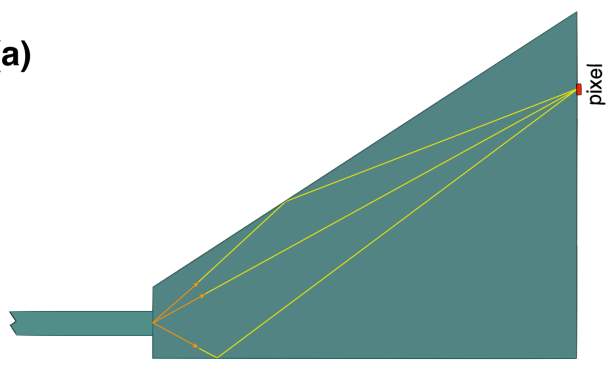

(b)

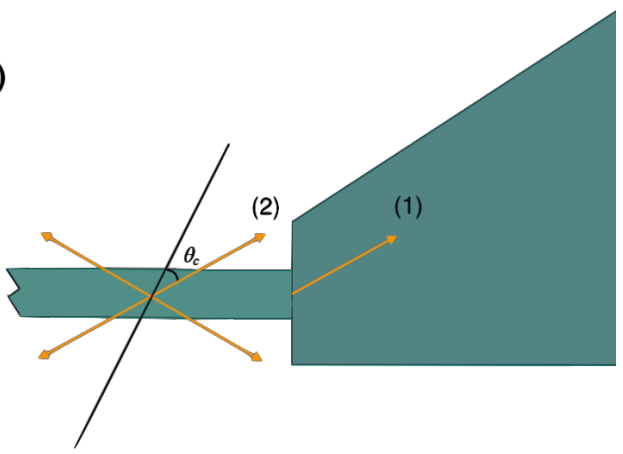

Figure 4. Schematic drawing of the geometrical reconstruction method. (a) All possible photon paths from the bar to the pixel (three examples are shown) are stored in look-up tables (LUT). (b) Close-up of the area of interest: the Cherenkov angle is determined by calculating the angle between the photon direction extracted from the LUT (1) and the charged track direction from the tracking system (2). Four of the eight possible ways to combine the photon vector with the track direction vector are shown.

The detected photon direction was approximated by a 3-D vector pointing from the end of the bar to the pixel center, including effects of refraction in the lens and reflections inside the prism, for every possible bar-pixel combination. The full Geant 4 simulation was used to simulate optical photons at the end of the bar and to track them until they were detected on the photo-sensor plane. For each bar-pixel combination the direction vectors were averaged and stored in a LUT (see figure 4a). The Cherenkov angle $\theta_{c}$ was calculated for each photon by combining the directions from the LUT with the charged particle direction vector (see figure 4b), taking all possible combinations into account. 


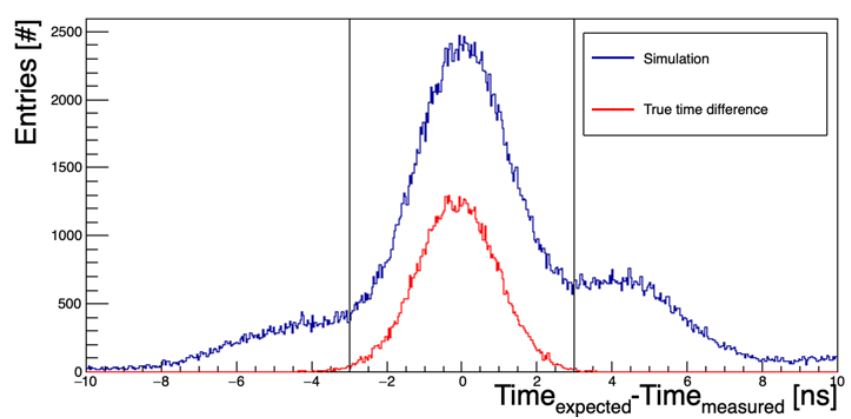

(a)

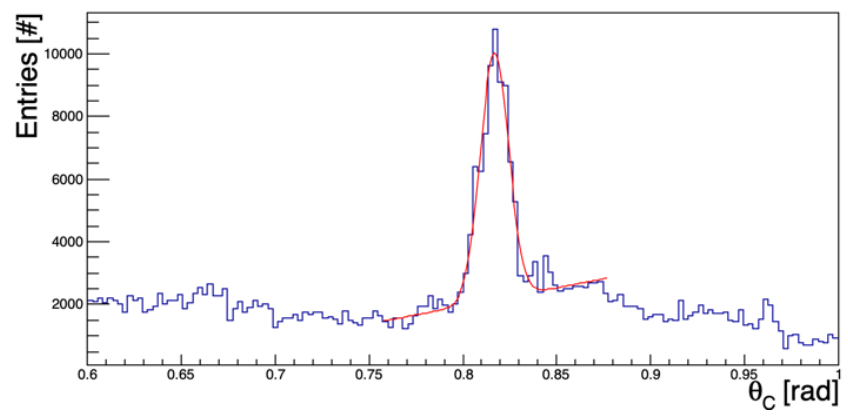

(b)

Figure 5. (a) Time difference between measured and expected arrival time of the Cherenkov photons from 1000 simulated protons from reconstruction (blue) and from Geant 4 truth information (red). The vertical lines indicate the selection cut. (b) Simulation of the reconstructed Cherenkov angle per photon from 1000 simulated protons with $7 \mathrm{GeV} / c$ momentum at $25^{\circ}$ polar angle. The fit result indicates that the Cherenkov angle resolution per photon is about $8 \mathrm{mrad}$.

Since the Cherenkov photons are internally reflected many times during propagation through the bar, the exact path of the photon is unknown, which in turn leads to ambiguous reconstructed photon directions. There are eight different possible direction combinations inside the bar (right/left, top/bottom, and forward/backward). They are taken into account by combining each possible photon direction vector with the beam direction vector, producing up to 8 different values for the reconstructed Cherenkov angle per photon. Further reconstruction ambiguities arise from the different possible reflections inside the prism, leading to 50 and more possible photon paths for some angles. The number of possible photon paths is reduced by discarding angles that are larger than the critical angle of fused silica and by requiring the Cherenkov angle of the reconstructed photon to be smaller than $1 \mathrm{rad}$. Additional reduction of this combinatorial background was achieved by applying a selection cut on the difference between the measured detection time of the photon and the expected detection time. The latter is the sum of the reconstructed photon path in the bar and the prism, calculated from the photon direction vector and the location of the particle track in the bar. The photon propagation time is calculated assuming a group velocity corresponding to the average wavelength of detected photons, which is $380 \mathrm{~nm}$, determined from simulation.

Figure 5a shows the time difference distribution for photons from 1000 protons with $7 \mathrm{GeV} / c$ momentum at $25^{\circ}$ polar angle in Geant4. Figure $5 \mathrm{~b}$ shows a simulation of the reconstructed 


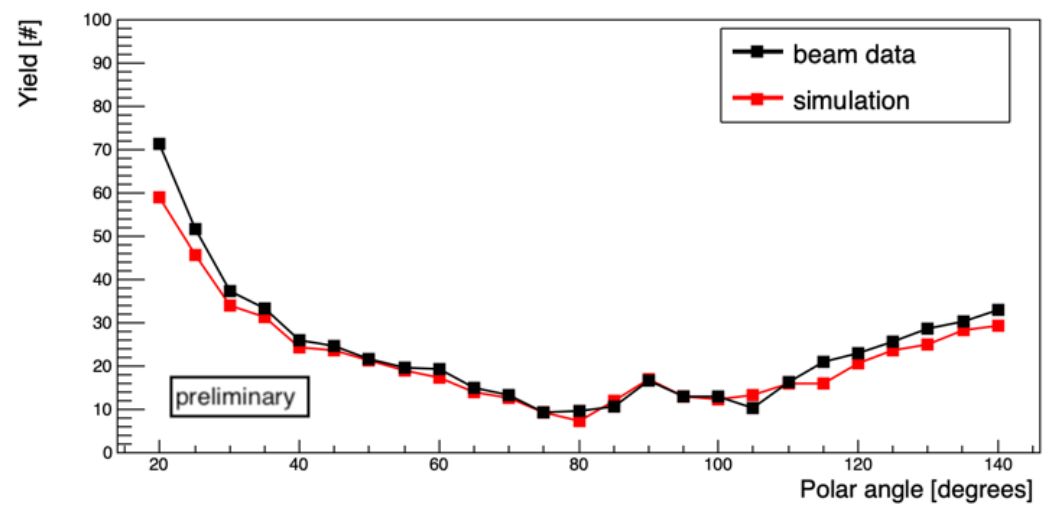

(a)

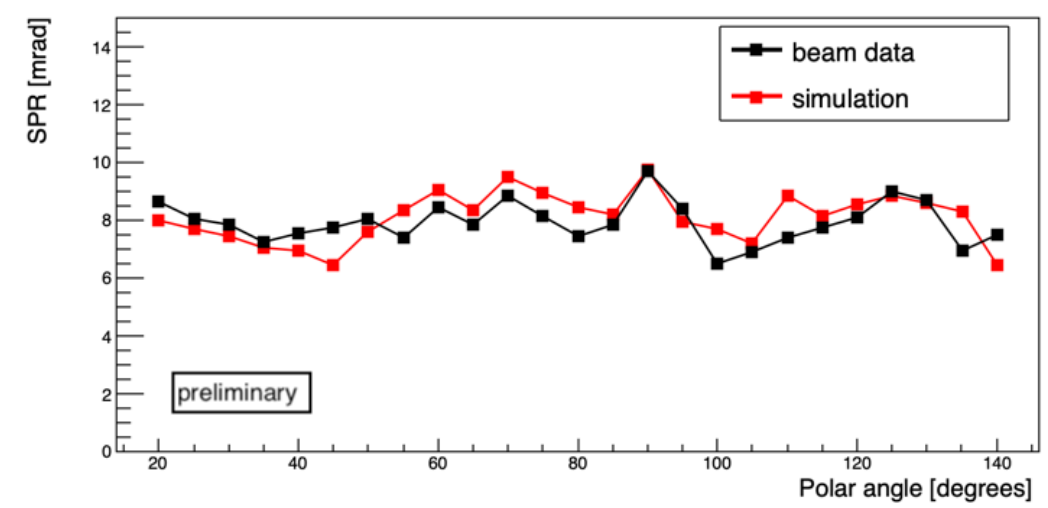

(b)

Figure 6. (a) Photon yield as a function of the prototype polar angle for tagged protons with $7 \mathrm{GeV} / \mathrm{c}$ momentum for beam data (black) and Geant4 simulation (red). (b) Cherenkov angle resolution per photon (SPR) as a function of the prototype polar angle for tagged protons with $7 \mathrm{GeV} / \mathrm{c}$ momentum for beam data (black) and Geant4 simulation (red).

Cherenkov angle per photon, including reconstruction ambiguities, for 1000 protons with $7 \mathrm{GeV} / c$ at $25^{\circ}$ polar angle. A clear peak can be observed at the expected value of the Cherenkov angle while the reconstruction ambiguities are visible as a linear background.

Misalignment effects were mitigated by applying a correction based on the average reconstructed Cherenkov angle per photon. The mean values of the reconstructed Cherenkov angle were extracted for each MCP-PMT from the fitting function across the prototype polar angle range. The fitted mean values were found to be shifted from the expected value, for both beam data and simulations, and to vary as a function of polar angle. The magnitude of the shift was calculated for each MCP-PMT and added as a correction to the Cherenkov angle per photon to bring the reconstructed angle in agreement with expectation.

Figure 6a shows the photon yield as a function of the prototype polar angle for both beam data and simulation. The single photon Cherenkov angle resolution (SPR) can be determined by fitting a Gaussian plus a linear background to the Cherenkov angle distribution per photon. The result is shown as a function of the polar angle in figure $6 \mathrm{~b}$. 


\section{Geometrical reconstruction}

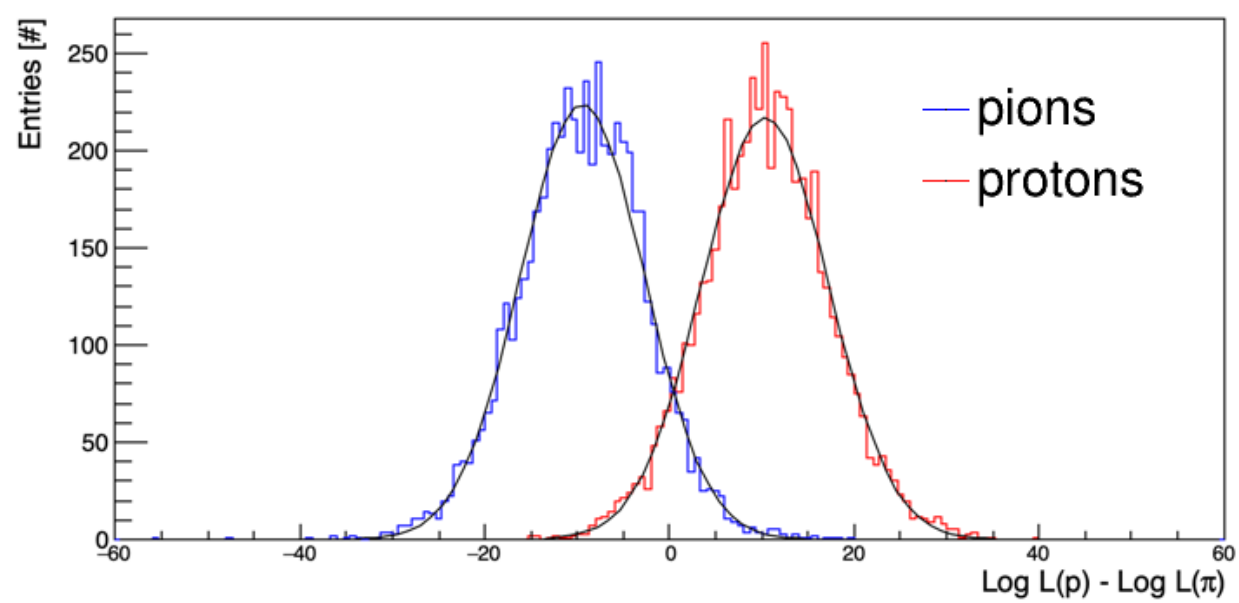

Figure 7. $\pi / \mathrm{p}$ log-likelihood difference distributions for proton-tagged (red) and pion-tagged (blue) beam events as result of the geometrical reconstruction at $7 \mathrm{GeV} / c$ momentum and a $25^{\circ}$ polar angle. The $\pi / \mathrm{p}$ separation power value is 2.9 s.d.

The distribution of the log-likelihood differences of the pion/proton hypothesis tests for the beam data with $7 \mathrm{GeV} / c$ and $25^{\circ}$ polar angle is shown in figure 7 . The distribution is fitted with two Gaussians and the $\pi / \mathrm{p}$ separation power is defined as the absolute value of the difference of the two mean values $\left(\mu_{1}, \mu_{2}\right)$ in units of the average of the two standard deviations $\left(\sigma_{1}, \sigma_{2}\right)$ s.d.,

$$
N_{\text {sep }}=\frac{\mu_{1}-\mu_{2}}{0.5\left(\sigma_{1}+\sigma_{2}\right)} .
$$

Figure 8 illustrates the $\pi / \mathrm{p}$ separation power from the proton-pion log-likelihood difference distributions for simulation and beam data as a function of the prototype polar angle.

\section{Geometrical reconstruction}

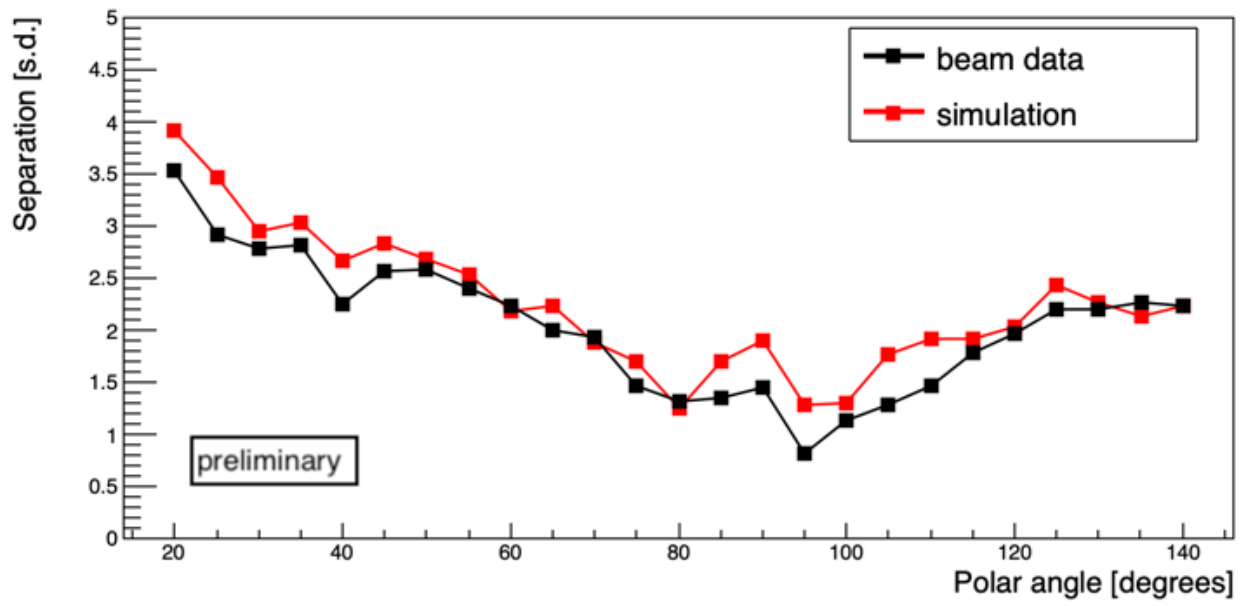

Figure 8. $\pi / \mathrm{p}$ separation power from geometrical reconstruction as a function of the polar angle at $7 \mathrm{GeV} / c$ momentum for beam data (black) and Geant4 simulation (red). 
The advantages of this reconstruction method is that it delivers measurements of the SPR, the Cherenkov angle of the charged particle track, and the photon yield, which are important for the detector performance evaluation. Moreover, the reconstruction algorithm is very fast since the LUTs can be created prior to event reconstruction and depend exclusively on the geometry of the detector and not on the charged particle properties.

\subsubsection{Cherenkov angle data PDF}

The basic concept is that the Cherenkov angle per photon is compared to the expected Cherenkov angle distributions for every pixel and every particle hypothesis, yielding the PID likelihoods. A large number of tracks from the beam data with a given momentum and polar angle was used to produce the expected Cherenkov angle distributions per photon for protons and pions. These distributions were recorded for every pixel and particle species and stored in an array of normalized histograms, creating probability density functions (PDF). For a given track, the observed Cherenkov angle per photon for each hit pixel is compared to the histogram array to calculate the Cherenkov angle PDF-based likelihood for the photons to originate from a certain particle hypothesis.

\section{Cherenkov angle data PDF}

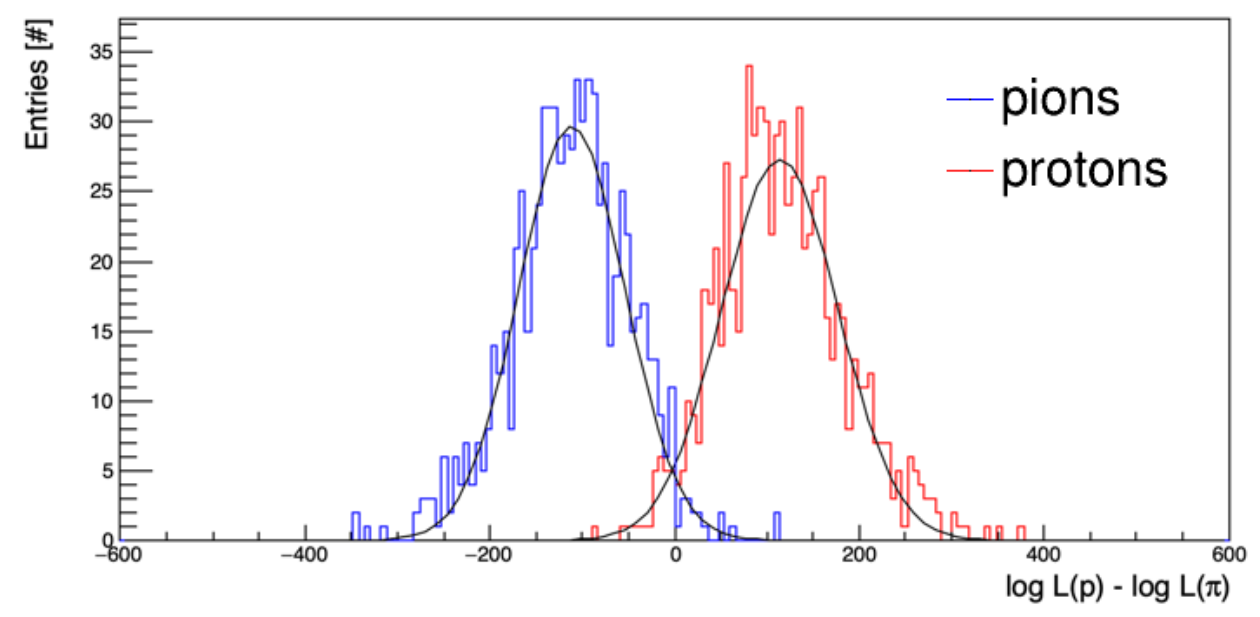

Figure 9. $\pi / \mathrm{p}$ log-likelihood difference distributions for proton-tagged (red) and pion-tagged (blue) beam events as result of the Cherenkov data PDF approach at $7 \mathrm{GeV} / c$ momentum and a $25^{\circ}$ polar angle.

Figure 9 shows the log-likelihood difference for tagged protons and pions with $7 \mathrm{GeV} / c$ at $25^{\circ}$ polar angle, with 3.7 s.d. pion/proton separation power. The $\pi / \mathrm{p}$ separation power is shown in figure 10 as a function of the polar angle at $7 \mathrm{GeV} / c$ momentum for beam data for three different reconstruction approaches: the standard geometrical reconstruction without corrections, the standard geometrical reconstruction after applying corrections, and the Cherenkov angle data PDF approach. The standard geometrical reconstruction improved after the corrections were applied, but the performance of the Cherenkov data PDF is still significantly better for most polar angles.

The main advantage of using the Cherenkov angle PDF from data is that it is much less dependent on proper alignment and calibration of the data, resulting in a better performance than the standard geometric reconstruction. The disadvantage is that a large number of tracks is required 


\section{Cherenkov angle data PDF}

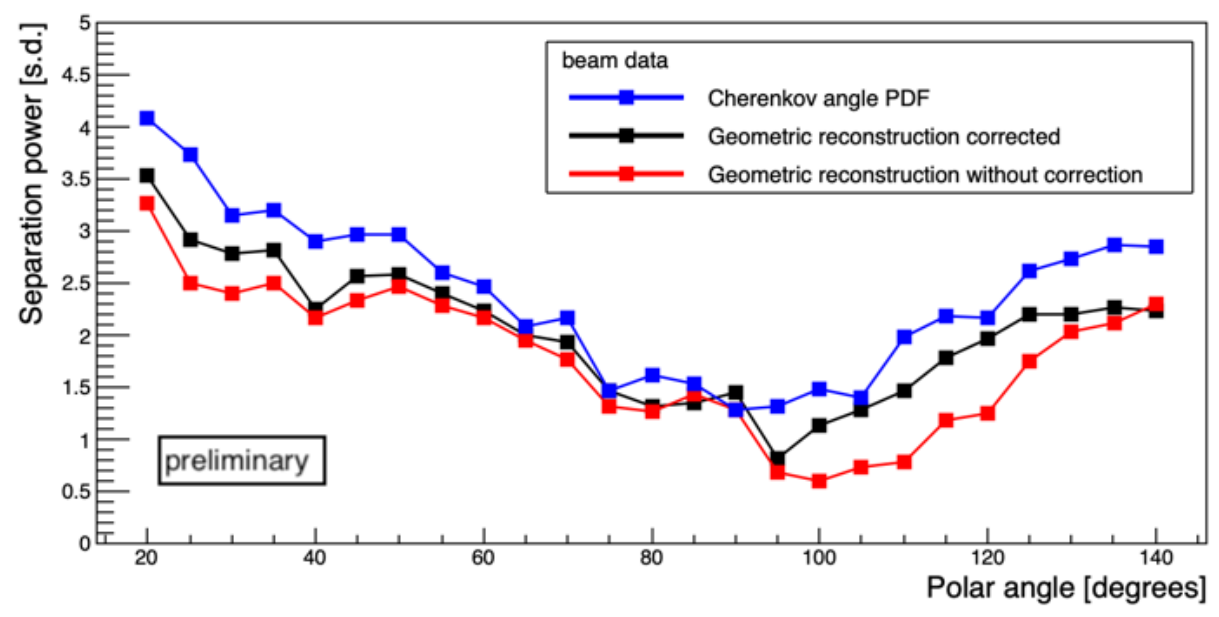

Figure 10. $\pi / \mathrm{p}$ separation power as a function of the polar angle at $7 \mathrm{GeV} / c$ momentum for beam data using: the geometrical reconstruction without corrections (red), the geometrical reconstruction after applying corrections (black), and the Cherenkov angle data PDF approach (blue).

for every track direction and momentum to create the PDFs. While this method works well for a beam test, it creates significant challenges for collider experiment data.

\subsection{Time-based imaging}

The time-based imaging method first was first developed for the Belle II time-of-propagation (TOP) counter [10]. The basic idea is that the measured detection time of Cherenkov photons is compared to the expected photon detection time for every pixel and particle hypothesis combination, resulting in PID likelihoods.

The expected photon detection times can be computed either analytically or from full simulation, or taken from the beam data itself. The latter approach was used to evaluate the PID of the time-based imaging approach for the PANDA Barrel DIRC prototype. The beam data PDFs are taken from a statistically independent sample with the same beam condition and detector configuration, separated by the TOF tags. Similarly, simulation PDFs are taken from a large number of simulated tracks with the same charged particle momentum and detector configuration. The detection time of the Cherenkov photons generated by protons and pions is stored for every pixel and saved in an array of normalized histograms to create the probability density functions (PDF). Figure 11 shows an example of the PDFs for protons and pions taken from beam data at $7 \mathrm{GeV} / c$ momentum and a polar angle of $25^{\circ}$ for two pixels in two different events.

Figure 12 shows the log-likelihood difference for proton and pion hypotheses for a sample with $7 \mathrm{GeV} / c$ protons and pions at $25^{\circ}$ polar angle. The calculated $\pi / \mathrm{p}$ separation power is $4.1 \mathrm{~s} . \mathrm{d}$. Figure 13 shows the $\pi / p$ separation power with $7 \mathrm{GeV} / c$ momentum as a function of the polar angle.

The main advantage of this reconstruction approach is that the performance is found to be superior to the geometrical reconstruction results. The disadvantages of the implementation using PDFs based on beam data or simulation are the large number of events required to create the PDFs and the large amount of computer memory required to store those PDFs. 


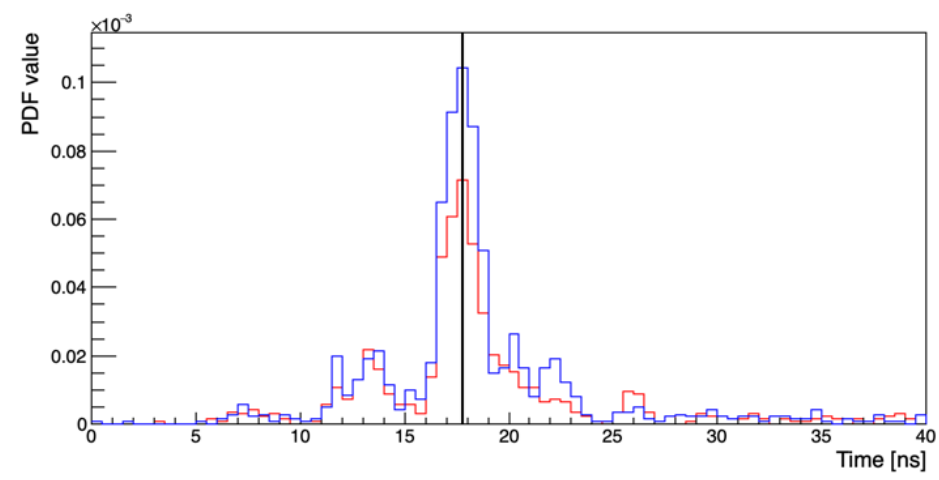

(a) Pion candidate

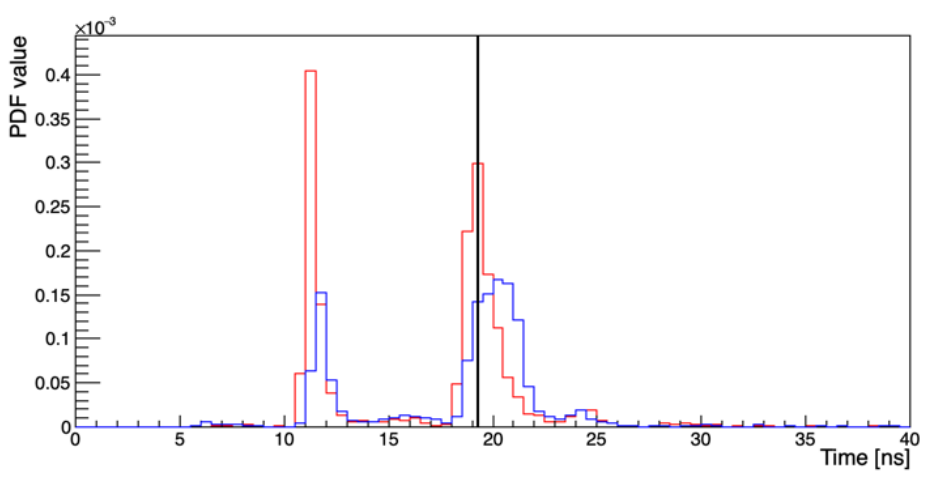

(b) Proton candidate

Figure 11. Example of the PDFs for two pixels for protons (red) and pions (blue) determined from beam data at $7 \mathrm{GeV} / c$ momentum and a polar angle of $25^{\circ}$ for two pixels. The vertical lines indicate the observed hit times for a pion candidate (a) and a proton candidate (b), based on the time-of-flight tag.

\section{Time-based imaging}

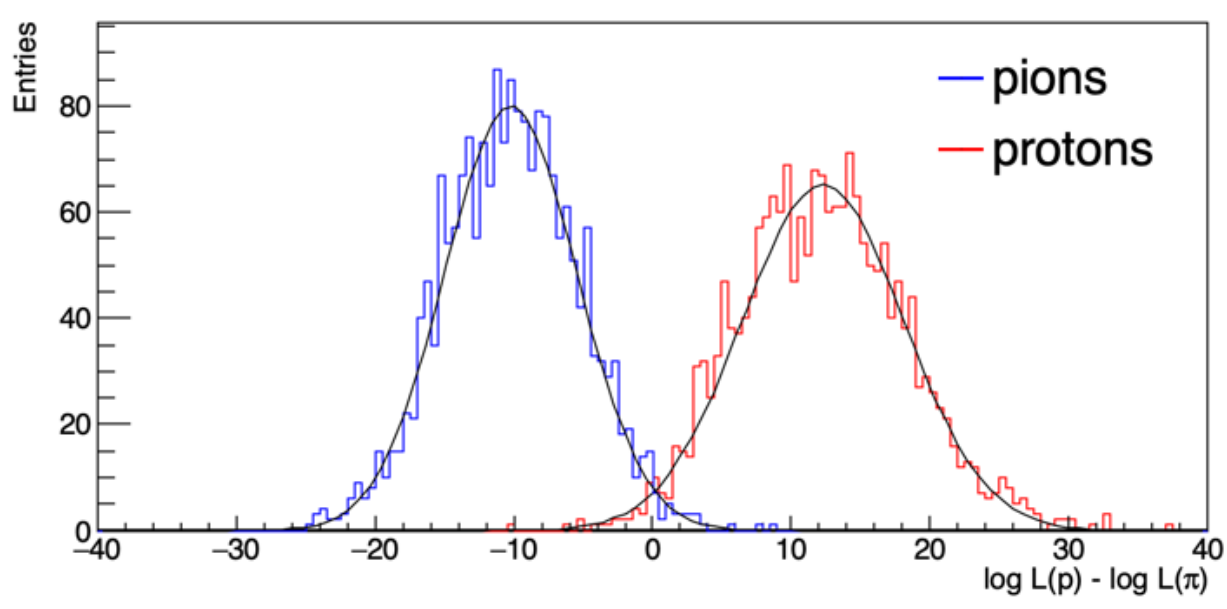

Figure 12. $\pi / \mathrm{p}$ log-likelihood difference distributions for proton-tagged (red) and pion-tagged (blue) beam events as a result of the time-based imaging reconstruction at $7 \mathrm{GeV} / c$ momentum and a $25^{\circ}$ polar angle. 


\section{Time-based imaging}

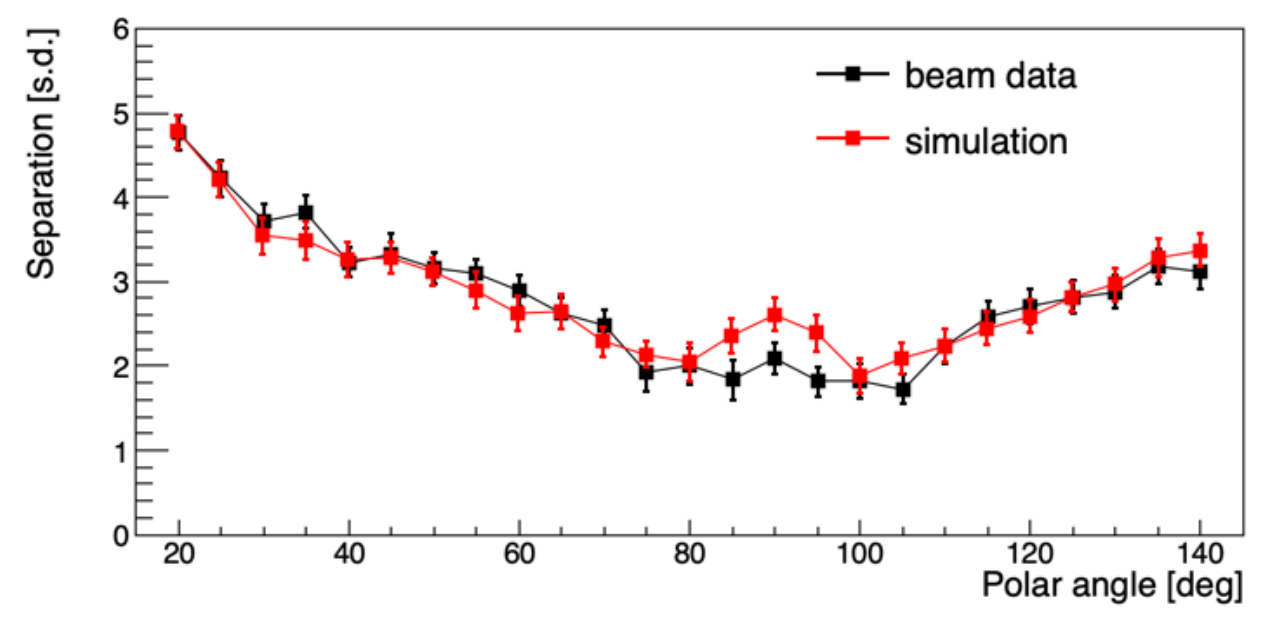

Figure 13. $\pi / \mathrm{p}$ separation power from time-based imaging as a function of the polar angle at $7 \mathrm{GeV} / c$ momentum the beam data (black) and the simulation (red) [11].

\subsection{Simplified time-based imaging}

This method is based on the time-based imaging reconstruction. It uses a simplified PDF creation approach, which significantly reduces the consumption of the computing resources. The full detector simulation was used to create the simplified time PDFs. The simulation stage started by virtually dividing the bar into segments; a point-like photon source was used to generate a large number of monochromatic photons at the center of the segment through which the track passed. Simulation information about the photon generation vector, the photon hit position on the photo-sensors, the photon arrival time, and the photon generation point are recorded and stored in a root file for further analysis. The expected photon detection time for every pixel and every particle hypothesis can then be calculated by applying a selection cut based on the Cherenkov angle per photon based on the particle momentum and hypothesis.

The arrival times of the photons are recorded for each pixel and stored in a normalized histogram array to create the PDF. The photon propagation times are smeared, depending on the photon path length, to account for the effect of the chromatic dispersion and the electronic readout timing resolution. The Cherenkov photon detection time is compared to the expected photon detection time for every pixel and particle hypothesis combination, resulting in PID likelihoods.

Figure 14 shows the log-likelihood difference for pions and protons with $7 \mathrm{GeV} / c$ momentum at $25^{\circ}$ polar angle for simulated events. The calculated $\pi / \mathrm{p}$ separation power is 3.5 s.d., which is about 0.6 s.d. less than the full time-based imaging method (see figure 13) but similar to the result for the Cherenkov data PDF method (see figure 10). Figure 15 shows the $\pi / \mathrm{p}$ separation power from the simplified time-based imaging for simulated events as a function of the polar angle with $7 \mathrm{GeV} / c$ momentum.

The main advantage of this method is that the PDFs can be generated for every particle hypothesis and momentum prior to event reconstruction. The disadvantages are that the approach still requires much more extensive computing resources than the geometric reconstruction or the analytical version of the time-based imaging. Furthermore, the performance is significantly worse 


\section{Simplified time-based imaging}

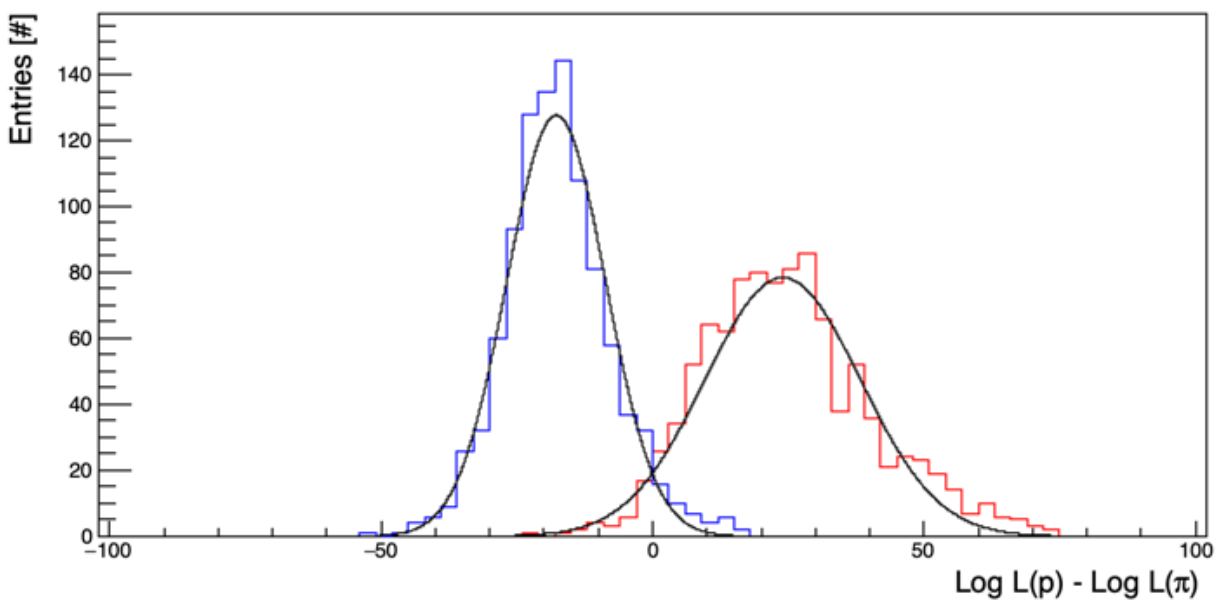

Figure 14. $\pi / \mathrm{p}$ log-likelihood difference distributions for protons and pions simulation events as a result of the simplified time-based imaging reconstruction at $7 \mathrm{GeV} / c$ momentum and a $25^{\circ}$ polar angle.

\section{Simplified time-based imaging}

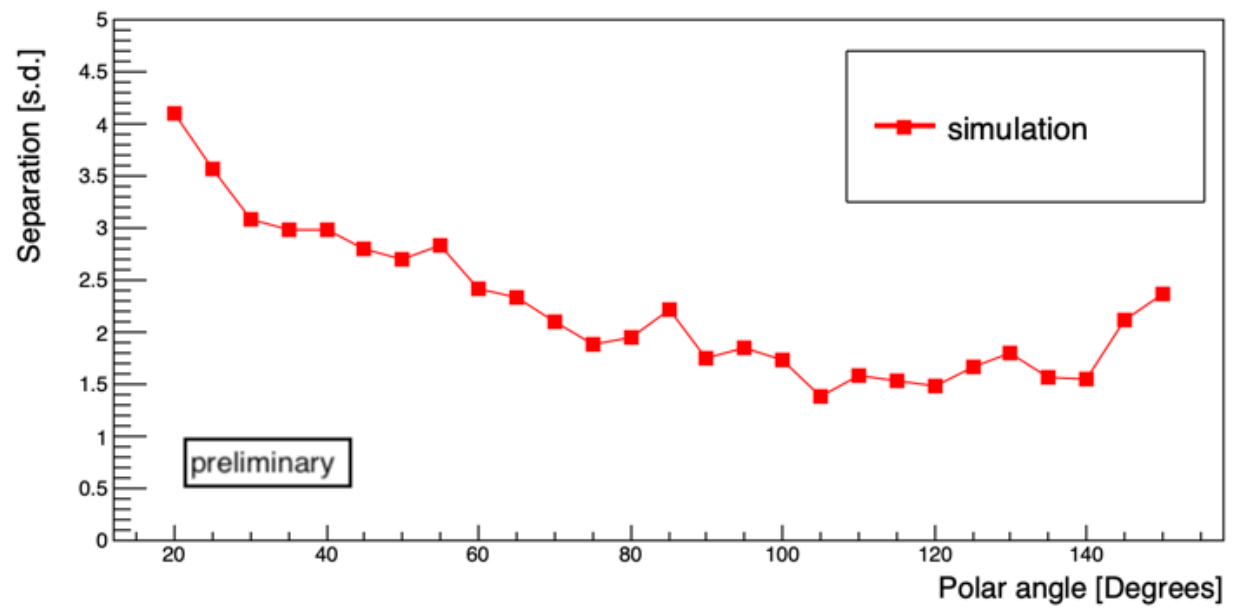

Figure 15. $\pi / \mathrm{p}$ separation power from simplified time-based imaging as a function of the polar angle at $7 \mathrm{GeV} / c$ momentum for simulation.

than the full time-based imaging method. This is due to a partial loss of the full Cherenkov angle and time information, caused by the simplifications and the selection cuts required to make this method faster and less demanding in terms of computing resources.

\section{Conclusion}

Several reconstruction algorithm approaches were developed for the PANDA Barrel DIRC. Geant4 simulations of the prototype and CERN PS test beam data were used to optimize and evaluate the performance of the algorithms. The geometrical construction delivers a measurement of the single photon Cherenkov angle resolution, the Cherenkov angle of the track, and the photon yield, which are essential variables for the DIRC detector performance studies. The geometrical construction is a 
fast algorithm since the LUTs can be created prior to event reconstruction. The time-based imaging reconstruction approach has a performance superior to the geometrical reconstruction, but requires large storage capacities for the PDFs for each angle, position, momentum, and particle type unless the PDFs can be calculated analytically. The analytical PDFs have not been implemented for PANDA yet and it remains to be seen if the performance is competitive with the results from the data PDFs shown here. The Cherenkov angle PDF method demonstrated encouraging results for $\pi / \mathrm{p}$ separation power as it performs better than the geometrical reconstruction. Furthermore, no additional correction is required as the detector misalignment effect was minimized. The disadvantage of the method is that large data samples are required to create the Cherenkov angle PDFs, which makes the approach more suitable to beam test experiments with a more restricted phase space and higher statistics. The simplified time-based imaging provides the time PDFs with a significantly reduced load on the computing resources. Initial results were promising, the PID performance is similar to the geometric reconstruction but is worse than for standard time-based imaging. Furthermore, the resource requirements are still rather demanding and need to be further optimized.

\section{Acknowledgments}

This work was supported by HGS-HIRe and HIC for FAIR. We thank the CERN staff for the opportunity to use the beam facilities and for their on-site support.

\section{References}

[1] J. Schwiening et al., The PANDA Barrel DIRC, 2018 JINST 13 C03004 [arXiv: 1803. 10642].

[2] B. Singh et al., Technical design report for the PANDA Barrel DIRC detector, J. Phys. G 46 (2019) 045001.

[3] PHOTONIS U.S.A. Pennsylvania Inc., 1000 New Holland Avenue, Lancaster, PA 17601-5688, U.S.A.

[4] Eljen Technology, 1300 W. Broadway, Sweetwater, Texas 79556, U.S.A.

[5] A. Neiser et al., TRB3: a 264 channel high precision TDC platform and its applications, 2013 JINST 8 C12043.

[6] Advanced Laser Diode Systems A.L.S GmbH, Schwarzschildstr. 6, D-12489 Berlin, Germany, https://www.nktphotonics.com/lasers-fibers/product/pilas-picosecond-pulsed-diode-lasers/.

[7] GEANT4 collaboration, GEANT4 - a simulation toolkit, Nucl. Instrum. Meth. A 506 (2003) 250.

[8] R. Dzhygadlo et al., Simulation and reconstruction of the PANDA Barrel DIRC, Nucl. Instrum. Meth. A 766 (2014) 263.

[9] I. Adam et al., The DIRC particle identification system for the BaBar experiment, Nucl. Instrum. Meth. A 538 (2005) 281.

[10] M. Starič, Pattern recognition for the time-of-propagation counter, Nucl. Instrum. Meth. A 639 (2011) 252.

[11] C. Schwarz et al., Status of the PANDA Barrel DIRC, 2020 JINST 15 C03055 [arXiv: 2004. 00913]. 